LA W RENCE LIVERMORE NATIONAL LABORATORY

\title{
Gravity Gradiometry: A Novel Application for Compressed Sensing
}

R. L. McNally

September 9, 2013 
This document was prepared as an account of work sponsored by an agency of the United States government. Neither the United States government nor Lawrence Livermore National Security, LLC, nor any of their employees makes any warranty, expressed or implied, or assumes any legal liability or responsibility for the accuracy, completeness, or usefulness of any information, apparatus, product, or process disclosed, or represents that its use would not infringe privately owned rights. Reference herein to any specific commercial product, process, or service by trade name, trademark, manufacturer, or otherwise does not necessarily constitute or imply its endorsement, recommendation, or favoring by the United States government or Lawrence Livermore National Security, LLC. The views and opinions of authors expressed herein do not necessarily state or reflect those of the United States government or Lawrence Livermore National Security, LLC, and shall not be used for advertising or product endorsement purposes.

This work performed under the auspices of the U.S. Department of Energy by Lawrence Livermore National Laboratory under Contract DE-AC52-07NA27344. 


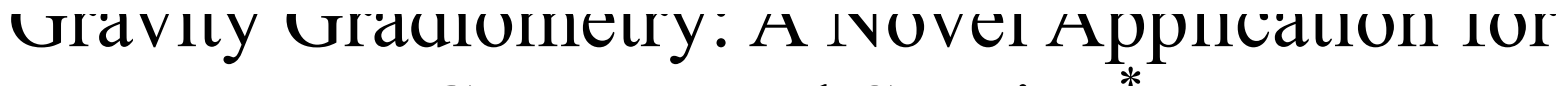 Compressed Sensing
}

\author{
Rees McNally ${ }^{\mathrm{a}}$
}

\begin{abstract}
During the past decade, compressed sensing (CS) has proven to be extremely useful for sparse signal reconstruction. Here the method of $\mathrm{CS}$ is applied to fast mass distribution determination based on cold atom gravity gradiometer measurements. Specifically we consider an array of $M$ gradiometers placed around a 2D target area in order to determine the interior mass distribution in cases where the set of (M) sensor measurements under samples the distribution. This was done by assuming that the system's sparsity comes from the mass distributions only having $K$ non-zero masses, which led to a non-orthogonal basis dictionary. This lack of orthogonality caused interesting behaviors, including weakened noise performance, and breaking of the typical CS motivated logarithmic scaling of required $M$ values for larger $K$ values. However, for low $\mathrm{K}$ values $\mathrm{M}$ scaled as expected. Modifications to the gravity sensor model to promote orthogonality and test its impact on signal recovery postponed the onset of anomalous scaling; suggesting that lack of orthogonality is the primary cause. While CS works for this sparse, but intrinsically ill-posed problem, this sensor system displayed increased noise sensitivity and a smaller upper bound on the size of recoverable $K$ sets. However, while these limitations decrease CS performance, significant improvements over traditional sensing approaches are still possible.
\end{abstract}

Index Terms-Atom Optics, Compressed Sensing, Signal Reconstruction, Sparse Representation

\section{INTRODUCTION}

Since its introduction, the framework of CS (Compressed S Sensing) has proven to be powerful in a wide range of applications. These have included a single pixel camera [1], MRI reconstruction [2], ADC design [3], biology [4] and geology [5]. In this work we will investigate how CS can be applied to a new form of non-invasive inspection, a gravity gradiometer portal based on modern cold atom interferometry [21]. Though this work is primarily focused on the specific gravimetric application, it is easily extended to other response limited inverse problems.

Since CS is based on the exploitation of signal sparsity, the problem of mass distribution determination ('gravity

${ }^{a}$ Rees L. McNally is at the University of Colorado, Boulder, CO, 80309, | USA. *LLNL-TR-643620. Work on this project was carried out at the Lawrence Livermore National Laboratory under DHS/DNDO contract CFP11-100-RTA-06-FP007, HSHQDC- 11-X-00550. Lawrence Livermore National Laboratory is operated by Lawrence Livermore National Security, LLC, for the U.S. Department of Energy, National Nuclear Security Administration under Contract DE-AC52-07NA27344. (e-mail: reeslmcnally@gmail.com). tomography') is a natural fit because many targets of interest will exhibit sparse deviations from expected background density patterns. For example the presence and localization of a block of dense material inside a crate is distinguished by relatively few extra variables from the background source of an empty crate. However, this is a non-traditional application for CS, as our sensor signal is not sparse in any orthogonal transform basis, but rather the system is sparse in the sources acted on by the gravity kernel. This feature poses several new challenges and interesting results emerge because gravity intrinsically lacks uniqueness, because different mass distributions can generate the same external gravitational field.

This paper will begin with a brief description of the signal model used for the gravity sensors. We will then outline the basic features of CS, with a focus on how they are implemented in this case. Finally, the impact of our problem's lack of sparsity in an orthogonal sense will be discussed, and its limitations considered.

\section{A. Sensor Design}

The sensors themselves are best understood by analyzing them as a series of steps, based on a semi-classical model. To begin with, a cloud of atoms is cooled and confined inside a MOT (Magneto-Optical Trap) [14-16]. Then the upper and lower confinement beams are detuned slightly, launching the cloud vertically in a fountain. As the cloud travels upwards it is hit with paired counter-propagating pulsed lasers driving the Raman effect, which splits the atomic wavepacket spatially into 2 parts, with correlated internal quantum numbers and momentum, that follow slightly different trajectories. At the peak of their trajectory the atoms are hit with another Raman pulse, which redirects the two wavepacket components towards each other. Lastly a third pulse completes the

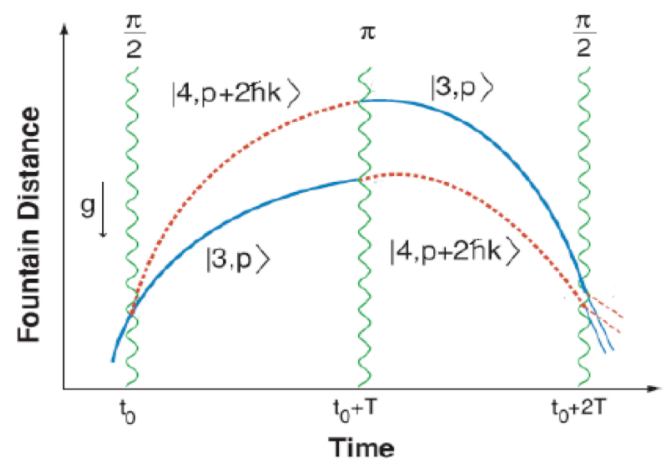

Fig. 1. Time-height plot of the atom cloud as it undergoes each Raman pulse. Momentum transfer from each Raman pulse is noted.

wavepacket reassembly. This process is shown in Fig. 1. [1719].

At the position where the atomic wavepacket re-combines the overall phase shift is measured via fluorescence. This entire process is effectively a measure of the difference in gravity seen along the two different trajectories. To reduce the 
impact of vibrational noise, the steps described above are done using two vertically seperated MOTs, whence the system operates as a gradiometer [20]. When this is all worked out, we are left with a linear relationship between the gravity gradient in the vertical direction, and the relative phase differences $\Delta \varphi$ between atoms in the two vertically separated atomic clouds at the three times and positions where Raman pulses occured [20].

$\nabla \mathrm{g} \propto\left[\Delta \varphi_{1}\left(\mathrm{t}_{0}\right)-2 \Delta \varphi_{2}\left(\mathrm{t}_{0}+\mathrm{T}\right)+\Delta \varphi_{3}\left(\mathrm{t}_{0}+2 \mathrm{~T}\right)\right]$ In a field with constant gravitational acceleration, the atoms in each cloud will experience the same effect from gravity, so their phases will all be equal. From (1) it follows that the gradient is zero, the expected result.

\section{B. Portal Configuration}

The general concept for the gravity portal is to augment current security radiation checkpoints, by identifying possibly shielded, anomalous masses in host vehicles passing through the gate [21]. This is an ill posed system, as there will be a finite number of sensors around the outside of the gate, attempting to determine the mass distribution inside. Furthermore, as already pointed out, the system is intrinsically ill-posed because different spherically symmetric mass distributions with the same total mass give identical external sensor readings.

For the purposes of this work, in order to simplify our computations, we will consider a $2 \mathrm{D}$ analog to the full portal sensor. Additionally we will limit the number of allowed mass locations, to the vertices of a grid. This is can be visualized as

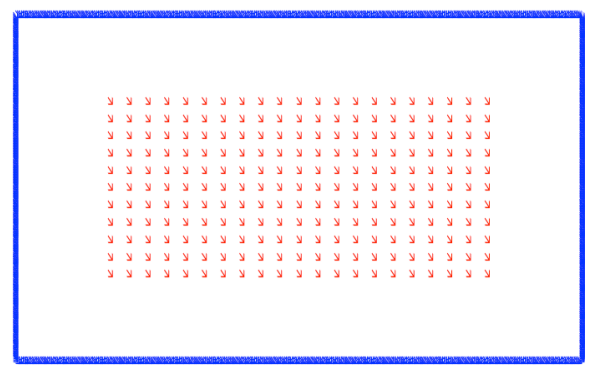

Fig. 2. Two dimensional security gate layout. Here red indices indicate potential mass locations, and the blue ring indicates potential sensor locations.

below.

Here the interior points show potential mass locations, and the exterior rectangle shows potential sensor locations. The sparsity condition for this setup comes from the assumption that only a few of the interior positions have nonzero masses at their location. This is an idealization of a car or shipping crate interior being predominately empty space, with a few added masses of interest.

An example of the resulting boundary gravity signals due to two distinct point masses is shown in Fig.3. It can be seen that small changes in mass positions will not result in large changes in the signal for certain areas of the portal. While which specific mass distributions are present, and the desired resolution of the gate are important factors in interpreting this fact, it is generally a problem that stems from gravity sensing, and cannot be avoided entirely.

\section{Compressed Sensing}

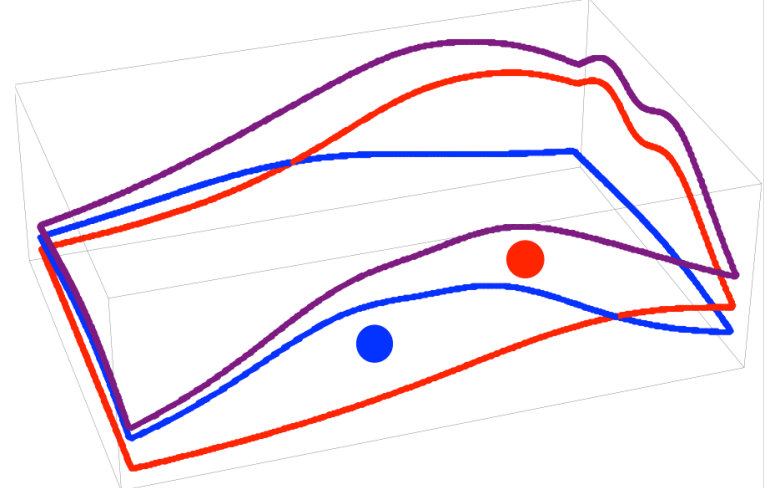

Fig. 3. Example signals caused by two (blue and red) point masses in the target region on the sensor ring. The total signal shown in purple is the summation of both of the individual elements,.

Mathematically, compressed sensing solves the equation $\mathrm{Y}=$ $\Phi S$, where $\mathrm{Y}$ is the measurement, $\mathrm{S}$ is the signal, and $\Phi$ is the recovery matrix. CS addresses the situation where $\mathrm{Y}$ is under sampled, meaning we have fewer samples than the ShannonNyquist requirement for full, general source recovery. For convention, let $\mathrm{M}$ (length of $\mathrm{y}$ ) be the number of measurements taken, and $\mathrm{N}$ (the length of $\mathrm{S}$ ) be the number of entries in the desired signal. Attempting to solve this system when $\mathrm{M}<<\mathrm{N}$ is then ill-posed. There is a large suite of tools for dealing with this problem by exploiting extra knowledge that might be available about S; CS exploits the knowledge that the signal $\mathrm{S}$ is 'sparse'. Here sparsity means that $\mathrm{S}$ has only K non-zero elements [8]. For this application this means there are only $\mathrm{K}$ non-zero point masses inside the gate.

So what exactly does knowledge of this sparsity enable? Consider again the system $\mathrm{Y}=\Phi \mathrm{S}$, but this time for a sparse vector S. Now, with Y being Mx1, $\Phi$ being $M x N$, and $\mathrm{S}$ being Nx1.This can be visualized as matrices, shown in Fig.4.

The fact that $\mathrm{S}$ is $\mathrm{K}$ sparse means it only has $\mathrm{K}$ non-zero

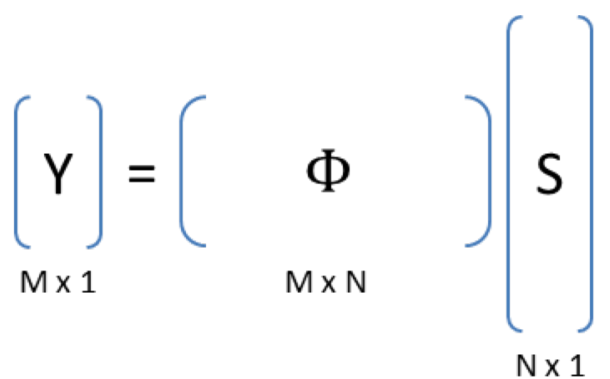

Fig. 4. Matrix visualization of the general CS problem setup. Dimensions are reflected in the relative shape of each matrix.

elements, so only K columns of $\Phi$ remain relevant after matrix multiplication. Which columns remain important depends on the positions of the nonzero entries. This selection process can be visualized using the same matrix shown in Fig. 5.

This effectively recasts the recovery problem where $\Phi^{*}$ is now $\mathrm{KxN}$, and $\mathrm{S}^{*}$ is $\mathrm{Kx} 1$. This new formulation is only an improvement if columns of the $\Phi^{*}$ matrix are orthogonal. So in order for the sparsity to prove useful, this must be true. This 


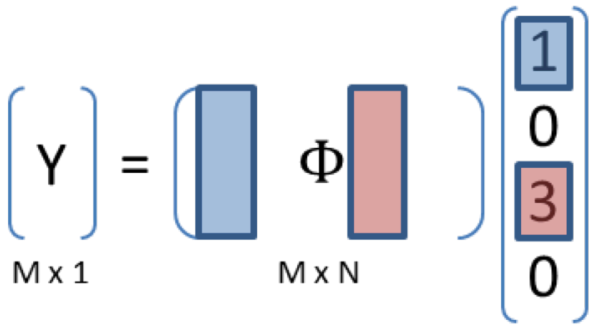

$\mathrm{N} \times 1$

Fig. 5. Color code shows which elements of $\Phi$ are selected.

property of $\Phi$ is known as the RIP (Restricted Isometry Property) - the requirement that each MxK sized sub-matrix of $\Phi$ be full rank [9].

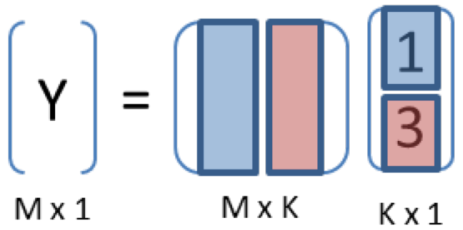

Fig. 6. The matrix representation of the recast problem. The MxK matrix here is $\Phi^{*}$. RIP requires that this matrix is well posed.

In general it has been shown that verification of the RIP is NP-hard [10]. However, if $\Phi$ is a random Gaussian matrix (or other sub Gaussian distribution) then to very high probability, $\Phi$ satisfies the RIP [8]. This is subject to a restriction on the number of measurements taken (M) shown in (2).

$M=O\left[k \log \left(\frac{N}{K}\right)\right]$

This is a very exciting result, as it shows that in principle sparse signals with very large $\mathrm{N}$ can be recovered readily by far fewer samples (M), which is exactly the desired behavior.

\section{Recovery Algorithms}

Now that we have the correct constraints to ensure that the problem properly utilizes the sparsity of $\mathrm{S}$, we must find an algorithm suitable for reconstruction. It turns out that the best recovery technique is based on the minimization of the L0 norm [8]. The L0 norm is an extensions of the LP norm for the case where $\mathrm{p}=0$, and functionally it counts the number of nonzero elements in a vector. For example the L0 norm of $\mathrm{S}$ is $\mathrm{K}$. With this knowledge, we might set out to solve the optimization problem, denoted by

$S^{*}=\operatorname{Min}|| S||_{0} \quad S . T . \quad Y=\Phi S$

However this algorithm is known to be NP-hard [12] for the same reason as RIP checking is NP-hard. This would seem to be a serious road-block. However if we relax the minimization problem to an L1 minimization, the results will be the same for most situations [8]. This means recovery can be achieved using the L1 relaxation of (3), which thankfully is computationally tractable.

$S^{*}=\operatorname{Min}|| S||_{1} \quad S . T . \quad Y=\Phi S$
Up until this point we have been assuming that the signal $\mathrm{S}$ is directly sparse, this is not generally true for all applications (though it is for some). A much larger class of problems have signals $\mathrm{X}$ that are sparse in some transformed basis $\Psi \mathrm{X}$. An example would be a signal consisting of $\mathrm{K}$ summed sinusoids, which is sparse under a discrete cosine transform. Expressed in matrix form this is the modified statement (5) that:

$S=\Psi X$ Therefore $Y=\Phi \Psi X$

This statement, while it does change the problem, can be viewed as a simple modification of the recovery matrix $\Phi^{*}=\Phi \Psi$. So the question now is whether $\Phi^{*}$ preserves all the desired properties of $\Phi$. This is only guaranteed to be true for $\Psi$ matrices corresponding to an orthogonal basis [8]. While this is a very useful result, here we wish to apply CS to a system (gravity) that does not have this orthogonality property.

\section{IMPLEMENTATION MODEL}

The setup for this problem is proposed as a general technique for applying CS to problems sparse in non-orthogonal frames. To highlight this, each component of the problem $(Y=$ $\Phi \Psi X$ ) will be discussed individually. The connections between the mathematical formulation and physical meaning will then be clear.

\section{A. $\Psi$, Sparse Basis Generation}

Considering that $\mathrm{X}$ must be sparse, we take values in $\mathrm{X}$ to represent both the locations and size of masses in the gate. This means that $\Psi$ maps the mass distribution into the sensed quantities. Stated differently, the $\Psi$ matrix characterizes (for a fixed sensor array) the set of all possible responses to mass source choices.

Thinking about the problem in this light $\Psi$ can be generated directly by calculating the response of the system to each potential stimulus. In this case we modeled the expected response for each of the sensors located along the outer ring of the gate, for each of the $\mathrm{N}$ interior mass locations. This means that a column of $\Psi$ is the response of each sensor to a single mass at a particular location, and a row of $\Psi$ is the response of a particular sensor, to a single mass at each of the potential locations. The sensor response for two particular mass locations, and the superposition of their gravity signals, is shown above in Fig.3. From equation (5) we see we have some flexibility with the dimensions of $\Psi$, so let it be PxN. For our application $\mathrm{P}$ is the total number of potential sensor locations along the sensing ring, but in general $\mathrm{P}$ can be thought of as the total number of potential ways the signal can be sampled. This is shown graphically in Fig.7

This concept of directly calculating the response to each potential stimulus is not unique to non-orthogonal dictionaries. For instance, consider a signal that is sparse in the Fourier domain, what would this method dictate $\Psi$ to be? It would be the signal magnitude at each time we are considering, for each of the possible frequencies. If phase information is not considered, then by generating $\Psi$ you would simply be constructing a DCT matrix. This provides an intuitive 


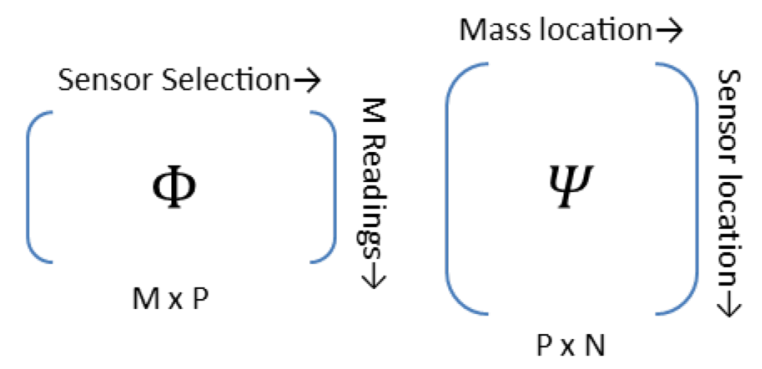

Fig. 7. Visualization for the physical interpretation for both $\Phi$ and $\Psi$. The $\mathrm{P}$ dimension noted here is the total number of potential sensor placements along the ring

interpretation for what a basis would be for a generalized CS application. This matrix is known as the dictionary in which the signal is sparse, and the system's response to each potential stimulus is known as an element in the dictionary.

\section{B. $\Phi$ Sensing/Collection Matrix}

Now we need to construct a $\Phi$ matrix that will both properly reflect the physical limitations of the system, and maintain the RIP. For the portal application, one consideration was the fact that we are limited to a chosen number of sensors. For a given limit of $\mathrm{L}$ sensors, and an implementation based on random 10 matrices, this means each row of $\Phi$ will have $L$ entries of value 1 , while the rest are 0 . This is true for each of the $M$ rows. For this work, $\mathrm{L}$ is approximately $1 \%$ of the $\mathrm{P}$ potential locations, amounting to using 11 sensors for each $\mathrm{M}$ measurement.

Physically what this means is that each 'measurement' takes the signal seen by L locations, randomly chosen around the sensor ring, and sums them. This is done $\mathrm{M}$ times, where each measure uses a different set of L. This shows how extra information is introduced into the system with CS. Though we only have $\mathrm{M}$ values coming out of the measurement cycle, each of these values in some way carries information for $\mathrm{L}$ locations. The $\Phi$ matrix contains the information on which sensor where used for each of the $\mathrm{M}$ measurements, and is used in reconstruction. Again, it is useful to keep in mind the representation of the $\Phi$ matrix shown in Fig.7.

When these two matrices are multiplied together it will give us the desired $\Phi^{*}$, as defined above. This is best thought of as a modified dictionary, based on the sensors that $\Phi$ selects from the full dictionary $\Psi$. For orthogonal $\Psi$ dictionaries reconstruction is possible using (4) subject to the limitations given in (2); however this is not guaranteed for the nonorthogonal $\Psi$ used in this application.

\section{RECOVERY ALGORITHM IMPLEMENTATION}

Now that we have defined how to generate each component of the matrices in (5), we must have a technique to solve for $\mathrm{X}$. There are many different algorithms currently used for recovery via L1 minimization. The two general classes of interest are greedy algorithms and linear programming. Neither of these methods is perfect, as each has pros and cons, and they are fundamentally limited by the RIP of the system.

\section{A. Greedy Algorithms}

The two most common forms of greedy algorithms used in CS recovery are BP (Basis Pursuit) [22] and OMP (Orthogonal Matching Pursuit) [23]. These are iterative fitting algorithms, where each iteration selects the single basis that minimizes the residual as much as possible. Said another way, these algorithms pick the best choice at each iteration, but do not find the globally best fit unless the two coincide.

These techniques are well suited for most situations with orthogonal dictionaries, as orthogonality ensures that each element is sufficiently different so that the locally correct choice is also the global choice [23].

However, for our application these algorithms perform very poorly for anything more than $\mathrm{K}=1$. In the case shown in Fig. 3 instead of returning the 2 separated masses, greedy algorithms recover a single larger mass between the two actual mass locations. Any two elements in this dictionary look a lot like another single element, because there are linearly dependent elements. This linear dependence is a mathematical manifestation of the lack of uniqueness in gravity signals.

\section{B. Linear Programming}

Because the L1 norm is simply the summation of every element of a vector, the minimization in (4) can be cast as a linear optimization problem. This enables solutions via an entire suite of algorithms. The performance of each was not specifically analyzed, but instead the stock Mathematica Linear Programming function was used. This package uses a blend of 'Revised Simplex' and 'Interior Point'. These algorithms search for the optimal $\mathrm{X}$ to minimize the global error in the reconstruction. For some orthogonal systems these algorithms are interchangeable with greedy algorithms, but for our purposes linear programming was the best option.

\section{RESUlTS}

The investigation of CS performance for this application took us down some unexpected lines of reasoning. To help facilitate overall understanding, different classes of results will be discussed individually, and then connected by shared behavior. The metric used for fit quality is the percentage of the recovered $\mathrm{X}$ values that are in the correct indices. Physically this corresponds to the percentage of the total mass placed in the correct locations.

\section{A. Sparsity Variation}

In order for CS to give an operational advantage, it must allow for reconstruction from a reduced (sub-Nyquist) number of measurements. This of course is also dependent on the degree of sparsity of the information, where the expected relationship is given by (2). Fig.8 illustrates how the recovery quality for the system approaches perfect recovery as a function of $\mathrm{M}$. 


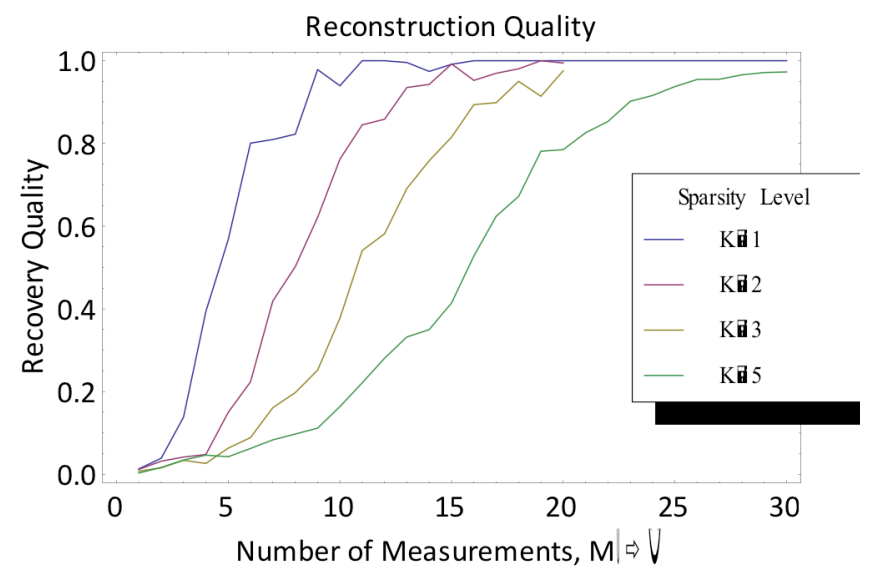

Fig.8. Convergence of reconstruction quality for low $\mathrm{K}$ sparsity levels. Here the decision for when to clip M is arbitrary.

As one can see there is a nice trend between sample count (M) and fit quality, which approaches perfect recovery as more samples are taken. Confirmation that the number of required measurements scales as (2) is shown by the number of measurements required for $80 \%$ reconstruction quality, for different $\mathrm{K}$ values in Fig.9.

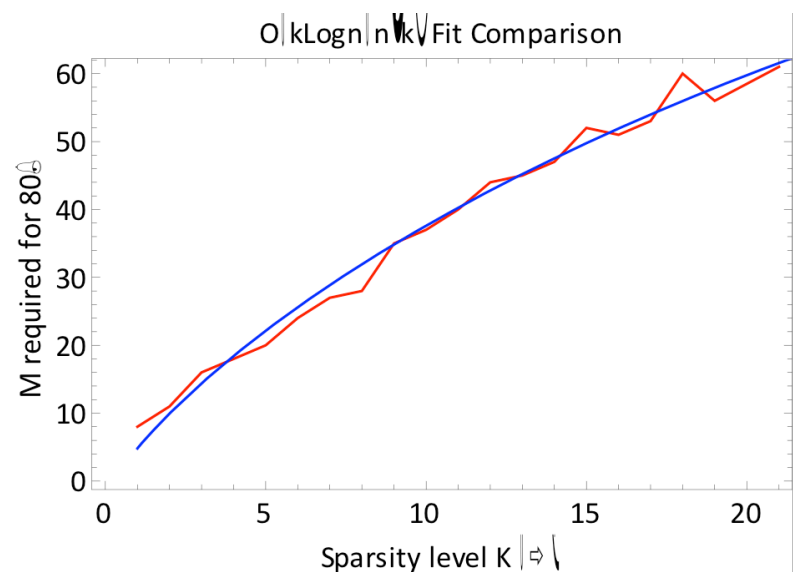

Fig. 9. $80 \%$ threshold, fitting to (2) showing that the required samples needed for reconstruction of various distributions scales as expected for low $\mathrm{K}$ values

This was implemented using 50 randomly generated $\mathrm{X}$ vectors, taking the average of the reconstruction quality for each, then increasing $\mathrm{M}$ until $80 \%$ average quality was achieved. The decision to not plot $\mathrm{K}$ greater than 21 in Fig.9 is not arbitrary, as the expected scaling breaks at that point. This asymptote at $\mathrm{M}=60$ represents a physical limit of the system, as for $\mathrm{M}$ larger than this, the algorithm begins to fail. This is very interesting because it is not predicted in classical CS, so intuitively it might be a product of the redundancy in the dictionary. That being said we see a nice agreement with the predicted scaling until the algorithm breaks.

This is an important result, as it shows that the degeneracies of the dictionary will limit performance of CS, but only for larger sparsity values. Therefore as long as one knows the limits placed on $\mathrm{K}$ for their system, in theory you can determine whether CS is a viable option or not. The degeneracies bound the maximal values of $\mathrm{M}$, which in turn bounds the size of $\mathrm{K}$ values.

\section{B. M Variation}

This limitation warrants closer examination. For constant sparsity levels, the fit quality was investigated as a function of $M$. If this was an orthogonal system, increasing $M$ past that value dictated by (2) would not give any better, or worse of a fit; it would simply converge to perfect recovery.

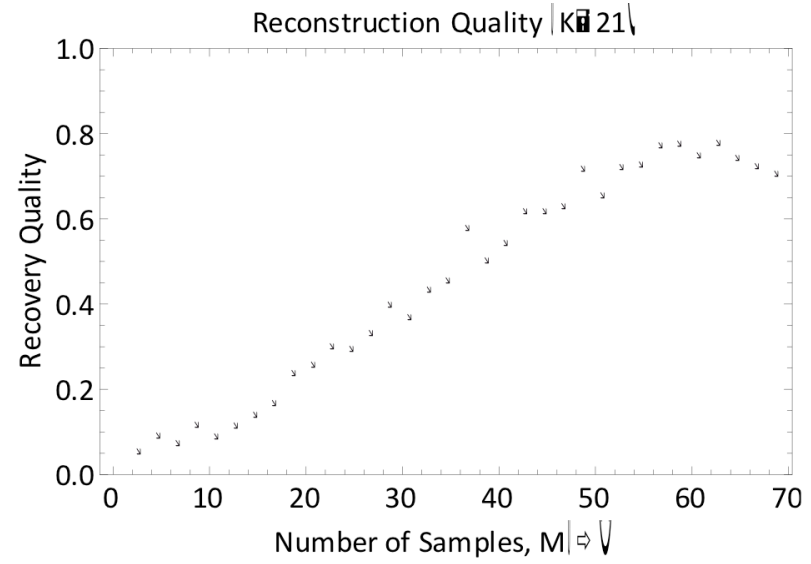

Fig. 10. High $\mathrm{K}$ sparsity recovery quality as a function of $\mathrm{M}$. While it would be expected to converge to $100 \%$ for large $\mathrm{M}$, instead we see degraded performance past a certain $\mathrm{M}$.

However we see a falloff in fit quality for high $\mathrm{M}$ values. This explains why for larger $\mathrm{M}$ levels the algorithm did not reach $80 \%$, because the fit quality decreases for higher M. In addition to this fall off, the algorithm begins to fail past $\mathrm{M}=70$. This was not expected, as there is no immediate way to see why introducing more data would give a worse fit. This is slightly reminiscent of over-fitting data. It would seem that qualitatively, we are reading too much into each measurement.

\section{Noise Behavior}

With this idea for why the algorithm fails, the noise performance was analyzed to see if any further clues could be found. Evidence for over-fitting comes when the scaling shown in Fig. 8 is re-calculated with 2 levels of additive Gaussian noise.

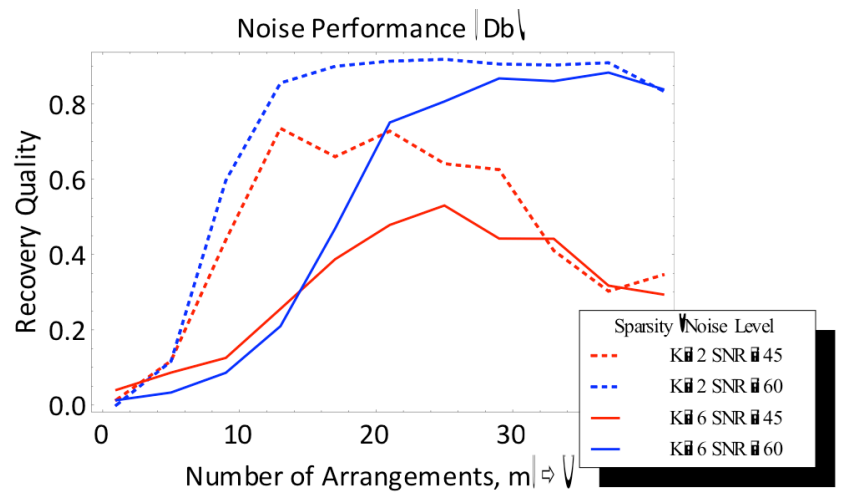

Fig. 11. The impact of noise on signal recovery scaling for low sparsity levels. SNR defined by the power of the signal, and the variance of additive Gaussian noise. SNR is approximate, as specifics depend on the mass distribution used for each of the samples averaged over.

In Fig. 11 we see that when noise is added, the M scaling roll-over begins at lower sparsity, and the systems performance is limited even more. This is important to recognize as it means that this extension of CS cannot be 
considered valid in general, as the source recovery is much more fragile in a dynamic noise environment.

\section{DICTIONARY REDUNDANCY EFFECTS}

At this point, while there is reason to believe that the unexpected behavior is due to the dictionaries redundancy, this has not been shown directly. By modifying the gravity kernel to be non-physical, yet less redundant, we can add evidence for this intuitive view. This was done by using a piecewise definition of gravity, allowing it to go as the inverse distance squared until it hits a certain threshold, then falling to zero. Here the degree of orthogonality is based on the distribution of each dictionary's singular values.

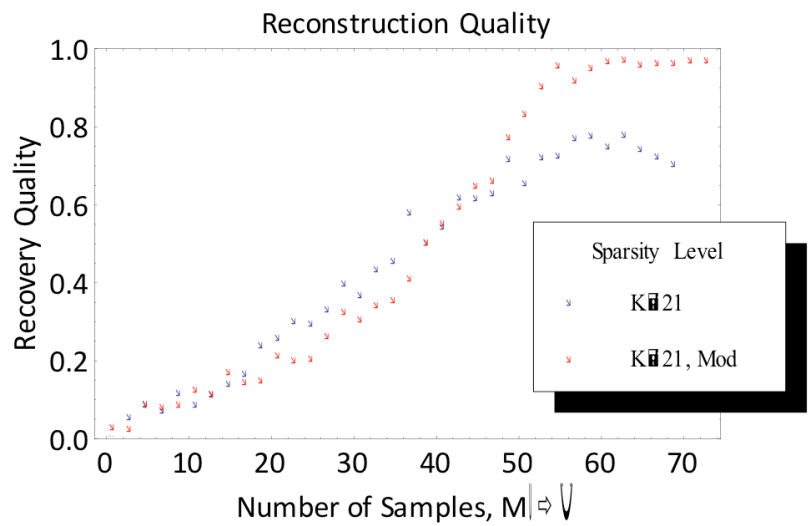

Fig. 12. Comparison of $\mathrm{M}$ convergence at fixed sparsity for two dictionaries. The red points used our modified dictionary constructed to have a larger degree of orthogonality.

As can be seen, the recovery quality roll-over behavior seen previously for the unmodified (physical) kernel is gone for this range of $\mathrm{M}$. Looking at the full range of $\mathrm{M}$, by re-analyzing how large an $\mathrm{M}$ we can get, and fitting to (2) we see that this

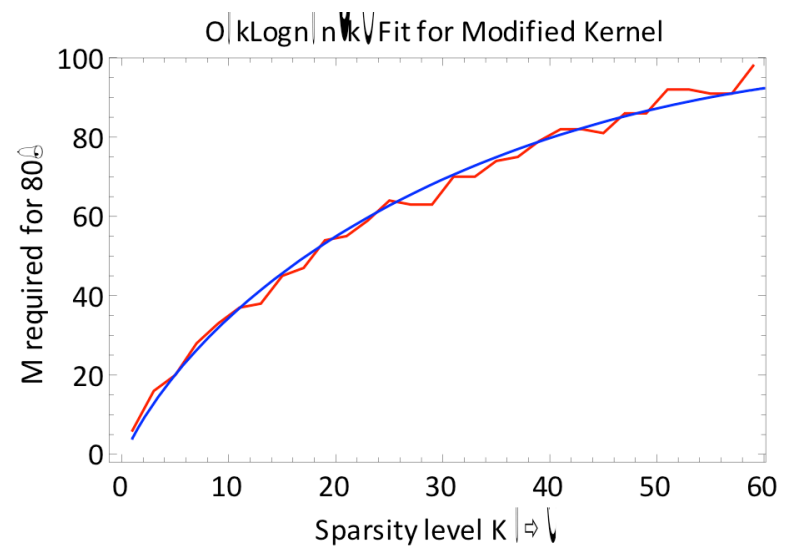

Fig. 13. This modified dictionary extends the validity of (2). However we still see point where the algorithm fails.

modified kernel does greatly improve fit quality.

This is quite an improvement, taking the tolerable range of sparsity from 21 to 60 , though the algorithm still begins to fail for $\mathrm{M}$ approximately equal to 90 . These trends extend to other modified dictionaries. Thus the degree of redundancy seems to be related to the valid range of $\mathrm{M}$ the algorithm can handle.

This also greatly improves noise performance, as seen in Fig.14. The modified system provides performance at $45 \mathrm{~dB}$ similar to the unmodified system at $60 \mathrm{~dB}$. In fact, we are able

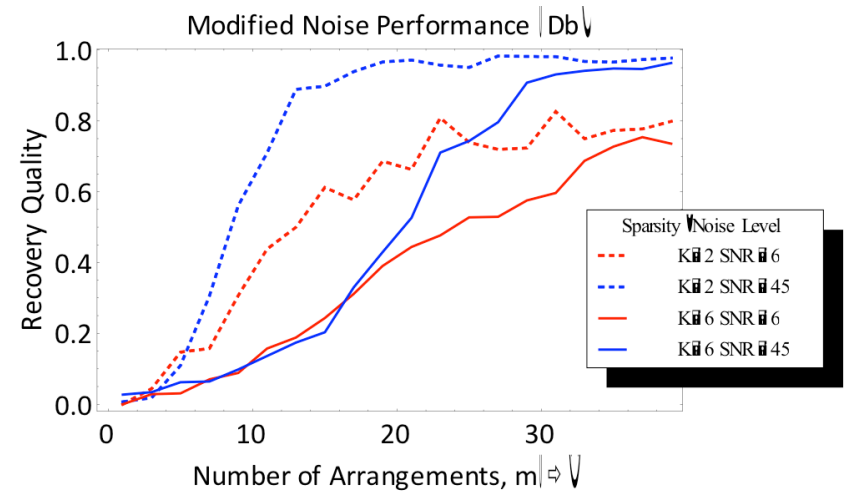

Fig. 14. Noise behavior for modified dictionary. Here we see that orthogonality of the dictionary impacts noise performance, in addition to $\mathrm{M}$ scaling.

to make meaningful reconstructions with only $6 \mathrm{~dB}$, something the unmodified dictionary was unable to do. This is further evidence that the degree of redundancy in the basis dictionary is the cause of the limitations on CS source recovery we discovered for the physical gravity sensor kernel.

\section{CONCLUSION}

Though it is a non-traditional application, we have successfully applied CS techniques to the reconstruction of mass distributions from gravity gradiometer measurements. This shows that the next generation of gravity gradiometers can be constructed to take advantage of these techniques, despite the lack of orthogonality requirements traditionally associated with CS. This opens up the design space, and may allow for truly novel designs in the future.

Additionally we have more precisely characterized the two primary limitations for applying CS to this type of nontraditional problem. These amount to limits on recoverable source sparsity, and increased in noise sensitivity. By artificially varying the gravity kernel we gave evidence that the lack of orthogonality in this problem's sparse dictionary is the primary source of the limitations. This implies that with proper modeling, new and interesting applications of CS can be explored, with potentially innovative results in unexpected fields.

\section{ACKNOWLEDGMENT}

I would like to thanks Dr. Stephen B. Libby, Dr. David H. Chambers, and Dr. Vijay Sonnad for suggesting this problem and for their guidance. Their experience provided insights and connections which were instrumental in the success of this work.

\section{REFERENCES}

[1] D. Takhar, V. Bansal , M. Wakin, M. Duarte, D. Baron, J. Laska, K. F. Kelly and R. G. Baraniuk, A compressed sensing camera: New theory and an implementation using digital micromirrors, Proc. Comput. Imaging IV SPIE Electronic Imaging, (2006).

[2] M. Lustig, D. Donoho and J. M. Pauly "Sparse MRI: The application of compressed sensing for rapid MR imaging", Magn. Reson. Med., vol. 58, pp.1182 -1195, (2007).

[3] S. Kirolos , J. Laska, M. Wakin, M. Duarte , D. Baron, T. Ragheb , Y. Massoud and R. G. Baraniuk, Analog-to-information conversion via 
random demodulation, Proc. IEEE Dallas Circuits Systems

Workshop, pp.71 -74, (2006).

[4] Vikalo, H.; Parvaresh, F. ; Hassibi, B., Signals, Systems and Computers, 2007. ACSSC 2007. Conference Record of the Forty-First Asilomar Conference, Page(s): 693 - 697, (2007).

[5] Lin, T. and Herrmann, F..,"Compressed wavefield extrapolation." GEOPHYSICS, 72(5), SM77-SM93. (2007).

[6] H. Rauhut, K. Schnass and P. Vandergheynst "Compressed Sensing and Redundant Dictionaries", IEEE Trans. Information Theory, 2007 E. P. Wigner, "Theory of traveling-wave optical laser," Phys. Rev., vol. 134, pp. A635-A646, Dec. 1965.

[7] Donoho, D.L. ; Elad, M. ; Temlyakov, V.N. Information Theory, IEEE Transactions on Volume: 52 , Issue: 1 Publication Year: 2006 , Page(s): $6-18$.

[8] D. L. Donoho "Compressed sensing", IEEE Trans. Inform. Theory, vol. 52, pp. $1289-1306,(2006)$

[9] R. G. Baraniuk "Compressive sensing", IEEE Signal Processing Mag., vol. 24, no. 4, pp.118-124, (2007).

[10] A. M. Tillmann, M. E. Pfetsch. (2012, May). The Computational Complexity of the Restricted Isometry Property, the Nullspace Property, and Related Concepts in Compressed Sensing [Online]. Available: arxiv.org.

[11] R. Baraniuk, M. Davenport, R. Devore, M. Wakin, "A simple proof of the restricted isometry property for random matrices," Constr. Approx, vol. 2008, (2007).

[12] M. Hyder and K. Mahata "An approximate 10 norm minimization algorithm for compressed sensing", Proc. Int. Conf. Acoustics, Speech, and Signal Processing (ICASSP), pp.3365 -3368, (2009).

[13] R. G. Baraniuk, V. Cevher, M. F. Duarte and C. Hegde "Model-based compressive sensing", IEEE Trans. Inf. Theory, vol. 56, no. 4, pp. 1982 -2001 (2010).

[14] Chu S, Bjorkholm J E, Ashkin A and Cable A Phys. Rev. Lett. 57, 314, (1986).

[15] Chu S, Hollberg L, Bjorkholm J E, Cable A and Ashkin A Phys. Rev. Lett. 55, 48, (1985).

[16] C. J. Bordé, Phys. Lett. A 140, 10, (1989),

[17] M. Kasevich and S. Chu, Phys. Rev. Lett. 67, 181 (1991).

[18] B. Young, M. Kasevich, and S. Chu, in Atom Interferometry, Academic Press, (1997).

[19] A. Peters, K. Y. Chung, S. Chu, Metrologia, 3, 25, (2001),

[20] J. M. McGuirk, G. T. Foster, J. B. Fixler, M. J. Snadden, and M. A. Kasevich, Phys. Rev. A 65, 0336008, (2002).

[21] S. B. Libby, V. Sonnad, S. A. Kreek, K. W. Brady, M. R. Matthews, B. Dubetsky, A. Vitouchkine, B. C. Young. Feasibility Study of a Passive, Standoff Detector of High Density Masses with a Gravity Gradiometer Based on Atom Interferometry, LLNL-TR-465878, (14 January 2011), Available: https://e-reports-ext.llnl.gov.

[22] S. Chen and D. Donoho "Basis pursuit", Proc. 28th Asilomar Conf. Signals, Syst. Comput., pp.41 -44, (1994).

[23] J.A. Tropp and A.C. Gilbert, "Signal Recovery from Partial Information via Orthogonal Matching Pursuit", preprint, April 2005. 